\title{
Criteria for Rational Use of Antibiotics in Over Aggregat- ed Bacterial Infection in Covid-19
}

\author{
Vásquez Cubas Humberto* \\ Infectology Unit, Hospital Grau Essalud, Lima, Perú
}

\begin{abstract}
The use of antibacterials, in almost all COVID cases that require hospitalization, has been widespread throughout the planet despite being a viral infection verified by molecular biology and tomographic images characteristic of bilateral atypical pneumonia. The WHO recommended using empirical antibiotic therapy "against all the most frequent pathogens" in cases of COVID pneumonia that require hospitalization. This abuse will have dire consequences on the bacterial flora due to selection pressure, mainly of extended spectrum beta-lactamase-producing Enterobacteriaceae, Pseudomonas aeruginosa, Acinetobacter baumanii and other multi-resistant bacteria. Some criteria or guidelines are proposed to decide or not the administration of antibacterials to cases of COVID with respiratory failure and need for hospitalization.
\end{abstract}

Keywords: COVID-19, Antibacterial agents, Standards

\section{Introduction}

At the beginning of the SARS COV-2 pandemic, medical personnel empirically established the administration of antibiotics in hospitalized patients, in such a way that with great frequency the vast majority of them received ceftriaxone and azithromycin under the presumption of a bacterial infection over-aggregated. The unnecessary administration of antibiotics alters the common resident flora, mainly of the pharynx and the colon of patients, exposes them to adverse effects, selects future resistance, increases the costs of care and does not improve the situation of those who have the viral infection. If the intention of administering antibiotics is to reduce mortality, the evidence shows that there was no significant impact. The systematic review with meta-analysis, published in August 2020 by Lei Pei et al. ${ }^{1}$ and Chedid Marie et.al ${ }^{2}$ evaluated retrospective studies that compared the use of antibiotics among survivors and non-survivors of Covid-19, that the Antibiotic use, in coronavirus infection 2019, had no impact on mortality.

Many COVID-19 Care Guidelines do not do not set out criteria for the use of antibiotics in the hyper-inflammatory phase; they limit themselves to recommending that these be used "when the doctor suspects concomitant bacterial infection" without specifying when to suspect with grounds and providing guidelines to better select the cases that justify the prescription or not of antibiotics. The World Health Organization recommends that patients with suspected or confirmed severe COVID-19 be administered empirical antibiotic treatment against all the most frequent pathogens. ${ }^{3}$

Indeed, after many months of the new coronavirus (SARS COV2) pandemic, no criteria have been proposed based on indicator patterns to suspect a concomitant or secondary bacterial infection in Covid-19 pneumonia. ${ }^{4} \mathrm{~A}$ meta-analysis of 30 studies with 3.834 observed cases has determined that, in patients hospitalized for COVID, the frequency of bacterial infection was $3.5 \%$ in patients admitted to hospitalization and up to $14 \%$ in those admitted to the ICU; however, more than $90 \%$ received antibiotics. ${ }^{5}$ Also, Lansbury et al. carried out a systematic review of what was published between January and April 2020, finding that only $7 \%$ of patients hospitalized with Covid had bacterial co-infection but that more than $90 \%$ received empirical antibiotic treatment. ${ }^{6}$ Timothy M Rawson et

\begin{tabular}{|l|l|}
\hline Quick Response Code: & *Corresponding author: Vásquez Cubas Humberto, Infectology Unit, Hospital Grau Essalud, \\
Lima, Perú \\
Received: 19 July 2021 \\
Citation: Humberto VC. Criteria for Rational Use of Antibiotics in Over Aggregated Bacterial \\
Infection in Covid-19. Curr Inv Cln Med Res. 2021;1(3):1-4. DOI: \\
10.53902/CICMR.2021.01.000514
\end{tabular}


al. ${ }^{7}$ determined that $8 \%$ of those hospitalized for Covid (62 of 806) had a bacterial or fungal infection during their hospitalization. ${ }^{7}$ Initially, the empiric use of antibiotics in hospitalized patients with Covid was justified, given the uncertainty. In the cases of respiratory syndrome due to the Middle East coronavirus, coinfection with bacteria in ICU patients was $18 \%{ }^{8}$ while with influenza the range of coinfections was between 11 and $35 \%{ }^{9}$ However, after more than a year of the Covid pandemic, the different epidemiological studies show that bacterial / Covid co-infection was demonstrated in less than $4 \%$ among hospitalized patients. ${ }^{10}$

\section{Development of the Topic}

The lived experience and the review of the main publications related to bacterial infections added to the Covid, has led us to propose the following systematization, proposing criteria to guide us on the need or not to use appropriate and timely antibiotics, avoiding their abuse. The extreme case of clear no justification is that with detection of SARS COV-2 by molecular test, radiological image characteristic of a viral process, with a normal blood count and absence of fever.

The evaluation of the following parameters: fever, time of illness, hemogram and procalcitonin level in the blood; they will allow the clinician in a sensible and empirical administration of antibiotics.

a) Fever is part of the viral picture, and can be present until the first days of the second week of infection. It often leads to the wrong decision to administer antibiotics despite the fact that it is not exclusive to the bacterial process. One of the first epidemiological reports evaluated 99 cases in January 2020, found that $83 \%$ of cases had fever, ${ }^{11}$ which is frequent in the first week and is not considered an alarm sign or criterion for hospitalization. The presentation of fever during the second week, in a hospitalized patient and after having remained afebrile, should be evaluated by investigating probable intrahospital infection.

b) It is necessary to evaluate the clinical context of each new case of Covid, considering the time of illness (from the onset of symptoms, not from the date of diagnosis). It is important to note that the bacterial complication occurs after the second week. In intensive care units, a multicenter retrospective cohort study found that secondary bacterial infection occurred in the vast majority of ICU patients between days 13 and $19 .{ }^{12}$

c) Another aspect to assess is the blood count, to observe the presence or not of leukocytosis ( $>12,000 x c u b i c m m)$. Like fever, leukocytosis is common both in bacterial and viral infection. On some occasions there may be transient neutropenia. An evaluation of hematological manifestations of patients with SARS due to coronavirus in Hong Kong, in 2003, ob- served lymphocytopenia in $98 \%$ of cases; while neutrophilia was present in $82 \% .^{13}$

d) Yajin Fu et al. found that $82.1 \%$ of cases with Covid infection had lymphocytopenia. ${ }^{14}$ Yang Liu, when correlating SARS COV2 viral load and lymphocyte counts, found that mild cases of Covid had an average of $1.21 \times 109$ (range: 0.99 to 1.6) and in severe cases the average was $0.45 \times 109$ (range: 0.3 to or.75). ${ }^{15}$

e) Procalcitonin production is greatly elevated during bacterial infections which is related to the concentrations of Intreleukin 1B, Tumor Necrosis Factor alpha and Interleukin 6. However, PCT synthesis is inhibited by increased interferon gamma during viral infections, which explains that its concentration does not rise much when there is uncomplicated SARS infection. ${ }^{16,17}$ The presence of low levels of procalcitonin has been shown to have a $94 \%$ negative predictive value for bacterial co-infection in Intensive Care Units in patients with confirmed influenza A H1N1. Therefore, the low values do not justify the administration of antibiotics in the patient with COVID-19. Low levels of procalcitonin, $<0.5 \mathrm{ng} / \mathrm{ml}$ have a high negative predictive value for bacterial infection. ${ }^{18-21}$

PCT $<0.1$ ug/L ATB strongly discouraged

PCT $<0.25-0.49 u g / L$ ATB advised against.

PCT $>0.5$ ATB recommended, according to clinical context

It is important to bear in mind those over-aggregated bacterial infections that occur in the hospital environment increase mortality. Patients hospitalized for Covid have bacterial complications with the common pathogens identified: Streptococcus pneumoniae, Haemophilus influenzae, Chlamydia pneumoniae, and Staphylococcus aureus; in these cases, betlactams such as ampicillin/ sulbactan or ceftriaxone plus macrolides or doxycycillin are recommended. ${ }^{22}$ These secondary infections are associated with invasive procedures, such as a mechanical ventilator, central venous catheter, urinary catheter, and feeding tubes. The study on secondary infections in a 3,000-bed hospital in New Delhi found that, among 1,179 hospitalized, $13 \%$ had positive cultures; the most frequent isolates were: Klebsiella pneumoniae (33: 3\%), Acinetobacter baumanii (27.1\%), Escherichia coli (16.7\%) and Pseudomonas aeruginosa $(11.5 \%),{ }^{23}$ the selection of antibiotics should be based on local resistance.

\section{Not everything is Infection}

It is important to consider that a patient whose evolution is unfavorable, after a recovery stage, without presenting criteria for the diagnosis of bacterial infection, may be developing a non-infectious process that is little identified and characterized by fatigue, cough, weight loss, crackles and elevation of inflammatory markers. ${ }^{24}$ In these cases, a new CT scan shows a predominance of consolida- 
tions, sometimes the inverted halo sign is present. ${ }^{25}$ This process is called organized pneumonia whose definitive diagnosis is histopathological by biopsy of the lung parenchyma with a finding of interstitial infiltration by myofibroblasts and collagen deposition, changes that can progress to fibrosis and obliteration of lung tissue. ${ }^{26} \mathrm{~A}$ bronchoalveolar lavage finding lymphocytes, neutrophils, and eosinophils will support the suspected diagnosis. If not everything is infection in these cases, then not everything is antibiotics, treatment with corticosteroids produces rapid improvement and prevents sequelae, although relapses are common when treatment is suspended and making it necessary to prolong treatment. ${ }^{27}$

Criteria for the prescription of antibiotics in patients with COVID pneumonia with detection of SARS COV-2 (molecular or antigenic test) and with bilateral radiological images with ground glass and / or crazy cobblestone appearance and / or consolidations:

1) Patient without fever and with normal blood count: do not prescribe antibiotics.

2) Patient with fever and or leukocytosis associated with lymphocytopenia, in the first days of respiratory failure, do not prescribe antibiotics: Repeat blood count and request procalcitonin. If hemogram is normal and procalcitonin is $<0.5 \mathrm{ng} /$ $\mathrm{ml}$ or fever remits spontaneously, maintain management without antibiotics.

3) History of bronchiectasis or chronic bronchitis, increased yellow or green sputum, supports suspicion of bacterial co-infection: prescribe antibiotic and request procalcitonin, repeat blood count after 24 hours. If procalcitonin is less than $0.5 \mathrm{ng} /$ $\mathrm{ml}$, stop antibiotics.

4) Patient who, after 48 hours hospitalized, being afebrile, starts or restarts fever and/or leukocytosis: request procalcitonin, blood cultures and blood count and start antibiotics.

5) Patient who, after remaining stable or improving, worsens during hospitalization, request new images, blood count and cultures and start antibiotics. Evaluate results to define whether to continue or not antibacterial.

6) Consider antibiotics when there is leukocytosis and/or fever, in the absence of confirmation of SARS COV-2 infection and the radiological image is not viral, especially if it is unilateral.

7) Consider the probability of organized pneumonia when the criteria for defining bacterial infection are insufficient or the response to treatment is not satisfactory.

\section{Acknowledgments}

None.

\section{Funding}

None.

\section{Conflicts of Interest}

Author declares that there is no conflict of interest.

\section{References}

1. Lei Pei, Sheng Zhang, Linxi Huang, et al. Antiviral agents, glucocorticoids, antibiotics, and intravenous immunoglobulin usage in 1142 patients with coronavirus disease 2019: a systematic review and meta-analysis. Pol Arch Intern Med. 2020;130(9):726-733.

2. Chedid Marie, Rami Waked, Elie Haddad, et al. Antibiotics in treatment of COVID-19 complications: a review off frequency, indications, and efficacy. J Infect Public Health. 2021;14:570-576.

3. WHO. Clinical management of COVID-19. Interim guidance; 2020.

4. Chao Ping Wu, Fatima Adhi, Kristin Highland. Recognition and management of respiratory coinfection and secondary bacterial pneumonia in patients with COVID-19. Cleve Clin J Med. 2020;87(11):659-663.

5. Bradley J Langford, Miranda So, Sumit Raybardhan, et al. Bacterial co-infection and secondary infection in patients with COVID19: a living rapid review and meta-analysis. Clin Microbiol Infect. 2020;26(12):1622-1629.

6. Lansbury Louise, Lim Benjamin, Vadsala Baskaran, et al. Co-infections in people with COVID-19: a systematic review and meta-analysis. J Infect. 2020;81(2):266-275.

7. Timothy M Rawson, Luke SP Moore, Nina Zhu, et.al. Bacterial and fungal co-infection in individuals with coronavirus: A rapid review to support COVID-19 antimicrobial prescribing. Clin Infect Dis. 2021;72(5):905-906.

8. Arabi YM, Al Omari A, Mandourah Y, et al. Critically Ill patients with the Middle East respiratory syndrome: a multicenter retrospective cohort study. Crit Care Med. 2017;45(10):1683-1695.

9. Klein EY, Monteforte B, Gupta A, et al. The frequency of influenza and bacterial coinfection: a systematic review and meta-analysis. Influenza Other Respir Viruses. 2016;10(5):394-403.

10. Thomas Lardaro, Alfred Z Wang, Antonino Bucca, et.al. Characteristics of COVID-19 patients with bacterial coinfection admitted to the hospital from the emergency department in a large regional healthcare system. Journal of medical Virology. 2021: 93(5):2883-2889.

11. Nanshan Chen, Min Zhou, Xuan Dong, et al. Epidemiological and clinical characteristics of 99 cases of 2019 novel coronavirus pneumonia in Wuhan, China: a descriptive study. Lancet. 2020;395:507-513.

12. Fei Zhou, Ting Yu, Ronghui Du, et.al. Clinical course and risk factors for mortality of adult inpatients with COVID-19 in Wuhan, China: a retrospective cohort study. Lancet. 2020;395(10229):1054-1062.

13. Raymond SM Wong, Alan Wu, To KF, et al. Haematological manifestations in patients with severe acute respiratory syndrome: retrospective analysis. BMJ. 2003;326(7403):1358-1362.

14. Yajing $\mathrm{Fu}$, Yuanxiong Cheng, Yuntao $\mathrm{Wu}$. Understanding SARS-CoV-2-Mediated Inflammatory Responses: From Mechanisms to Potential Therapeutic Tools. Virologica Sinica. 2020;35(3):266-271.

15. Yang Liu, Wenjian Liao, Lagen Wan, et al. Correlation Between Relative Nasopharyngeal Virus RNA Load and Lymphocyte Count Disease Severity in Patients with COVID-19. Viral Immunology. 2021;34(5):330335.

16. Giuseppe Lippi, Mario Plebani. Procalcitonin in patients with severe coronavirus disease 2019 (COVID-19): a meta-analysis. Clinica Chimica Acta. 2020;505:190-191.

17. Han jennie, Gatheral Timithy, Williams Craig. Procalcitonina para estratificación de pacientes e identificación de co-infección bacteriana en COVID-19". Clin Med (Lond). 2020;20(3):e47. 
18. Broyles Michael. Impact of Procalcitonin-Guided Antibiotic Management on Antibiotic Exposure and Outcomes: Real-world Evidence. Open Forum Infectious Diseases. 2017;4(4):ofx213.

19. Michael May, Michelle Chang, Donald Dietz, et al. Limited Utility of Procalcitonin in Identifying Community-Associated Bacterial Infections in Patients Presenting with Coronavirus Disease 2019. Antimicrob Agents Chemother. 2021;65(4):e02167-e02220.

20. Peters Christina, Williams Kelly, Elena A Un, et al. Use of procalcitonin for antibiotic stewardship in patients with COVID-19: A quality improvement project in a district general hospital. Clinical Medicine. 2021;21(1):e71-e76.

21. Philipp Schuetz, Werner Albrich, Beat Mueller. Procalcitonin for diagnosis of infection and guide to antibiotic decisions: past, present and future. BMC Medicine. 2011;9:107.

22. Joshua P Metlay, Grant W Waterer. Treatment of Community-Acquired Pneumonia During the Coronavirus Disease 2019 (COVID-19) Pandemic. Ann Intern Med. 2020;173(4):304-305.
23. Surbhi Khurana, Parul Singh, Neha Sharad, et al. Profile of co-infections \& secondary infections in COVID-19 patients at a dedicated COVID-19 facility of a tertiary care Indian hospital: Implication on antimicrobial resistance. Indian J Med Microbiol. 2021;39(2):147-153.

24. Sveinsson OA, Isaksson HJ, Sigvaldason A, et al. Clinical features in secondary and cryptogenic organising pneumonia. Int J Tuberc Lung Dis. 2007;11:689-694.

25. Ribeiro de Sales, et al: The Reversed Halo Sign and la COVID-19: Possible Histopathological Mechanisms Related to the Appearance of This Imaging Finding. Arch Bronocneum. 2021;57(S1):70-96.

26. Pierre Kory, Jeffrey Kanne. SARS-CoV-2 organising pneumonia: 'Has there been a widespread failure to identify and treat this prevalent condition in COVID-19?'. BMJ Open Resp Res; 2020.

27. JF Cordier. Cryptogenic organising pneumonia. Eur Respir J. 2006;28:422-446. 